AperTO - Archivio Istituzionale Open Access dell'Università di Torino

\title{
A balloon-borne prototype for demonstrating the concept of JEM-EUSO
}

\section{This is a pre print version of the following article:}

Original Citation:

Availability:

This version is available http://hdl.handle.net/2318/156050

since

Published version:

DOI:10.1016/j.asr.2013.11.049

Terms of use:

Open Access

Anyone can freely access the full text of works made available as "Open Access". Works made available under a Creative Commons license can be used according to the terms and conditions of said license. Use of all other works requires consent of the right holder (author or publisher) if not exempted from copyright protection by the applicable law. 


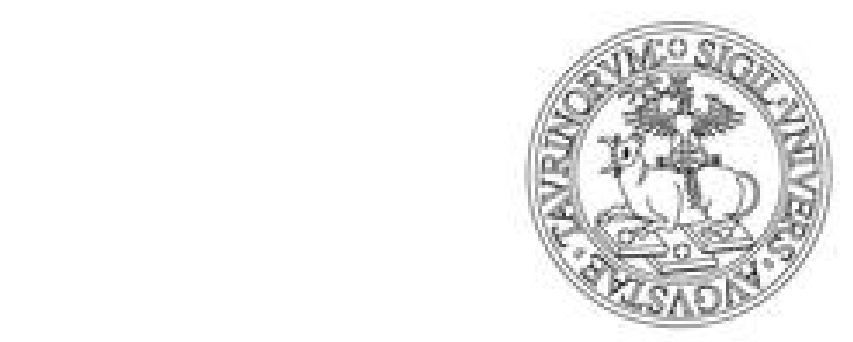

\section{UNIVERSITÀ DEGLI STUDI DI TORINO}

This Accepted Author Manuscript (AAM) is copyrighted and published by Elsevier. It is posted here by agreement between Elsevier and the University of Turin. Changes resulting from the publishing process - such as editing, corrections, structural formatting, and other quality control mechanisms - may not be reflected in this version of the text. The definitive version of the text was subsequently published in ADVANCES IN SPACE RESEARCH, 53, 2014, 10.1016/j.asr.2013.11.049.

You may download, copy and otherwise use the AAM for non-commercial purposes provided that your license is limited by the following restrictions:

(1) You may use this AAM for non-commercial purposes only under the terms of the CC-BY-NC-ND license.

(2) The integrity of the work and identification of the author, copyright owner, and publisher must be preserved in any copy.

(3) You must attribute this AAM in the following format: Creative Commons BY-NC-ND license (http://creativecommons.org/licenses/by-nc-nd/4.0/deed.en), 10.1016/j.asr.2013.11.049

The definitive version is available at:

http://dx.doi.org/10.1016/j.asr.2013.11.049 


\title{
A balloon-borne prototype for demonstrating the concept of JEM-EUSO
}

P. von Ballmoos ${ }^{1}$, A. Santangelo ${ }^{5}$, J.H. Adams ${ }^{19}$, P. Barrillon ${ }^{2}$, J. Bayer ${ }^{5}$, M. Bertaina ${ }^{13}$, F. Cafagna ${ }^{7}$, M. Casolino ${ }^{14,11,12}$, S. Dagoret ${ }^{2}$, P. Danto ${ }^{4}$, G. Distratis ${ }^{5}$, M. Dupieux ${ }^{1}$,

A. Ebersoldt ${ }^{6}$, T. Ebisuzaki ${ }^{14}$, J. Evrard ${ }^{4}$, Ph. Gorodetzky ${ }^{3}$, A. Haungs ${ }^{6}$, A. Jung ${ }^{15}$, Y. Kawasaki ${ }^{14}$, G. Medina-Tanco ${ }^{16}$, B. Mot $^{1}$, G. Osteria ${ }^{9}$, E. Parizot ${ }^{3}$, I.H. Park ${ }^{15}$, P. Picozza ${ }^{14,11,12}$, G. Prévôt ${ }^{3}$, H. Prieto ${ }^{18}$, M. Ricci $^{8}$, M.D. Rodríguez Frías ${ }^{18}$, G. Roudil ${ }^{1}$, V. Scotti ${ }^{9,10}$, J. Szabelski ${ }^{17}$, Y. Takizawa ${ }^{14}$, K. Tsuno ${ }^{14}$, for the JEM-EUSO Collaboration ${ }^{20}$

${ }^{1}$ Institut de Recherche en Astrophysique et Planétologie, Toulouse, France

${ }^{2}$ Laboratoire de l'Accélérateur Linéaire, Univ Paris Sud-11, CNRS/IN2P3, Orsay, France

${ }^{3}$ AstroParticule et Cosmologie, Univ Paris Diderot, CNRS/IN2P3, Paris, France

${ }^{4}$ Centre National d'Etudes Spatiales, Centre Spatial de Toulouse, France

${ }^{5}$ Institute for Astronomy and Astrophysics, Kepler Center, University of Tübingen, Germany

${ }^{6}$ Karlsruhe Institute of Technology (KIT), Germany

${ }^{7}$ Istituto Nazionale di Fisica Nucleare - Sezione di Bari, Italy

${ }^{8}$ Istituto Nazionale di Fisica Nucleare - Laboratori Nazionali di Frascati, Italy

${ }^{9}$ Istituto Nazionale di Fisica Nucleare - Sezione di Napoli, Italy

${ }^{10}$ Università di Napoli "Federico II" - Dipartimento di Scienze Fisiche, Napoli, Italy

${ }^{11}$ Istituto Nazionale di Fisica Nucleare - Sezione di Roma Tor Vergata, Italy

${ }^{12}$ Università di Roma Tor Vergata - Dipartimento di Fisica, Roma, Italy

${ }^{13}$ Dipartimento di Fisica dell' Università di Torino and INFN Torino, Torino, Italy

${ }^{14}$ RIKEN Advanced Science Institute, Wako, Japan

${ }^{15}$ Sungkyunkwan University, Suwon-si, Kyung-gi-do, Republic of Korea

${ }^{16}$ Universidad Nacional Autónoma de México (UNAM), Mexico

${ }^{17}$ National Centre for Nuclear Research, Lodz, Poland

${ }^{18}$ Universidad de Alcalá (UAH), Madrid, Spain

${ }^{19}$ University of Alabama in Huntsville, Huntsville, USA

${ }^{20}$ http://jemeuso.riken.jp

\begin{abstract}
EUSO-BALLOON has been conceived as a pathfinder for JEM-EUSO, a mission concept for a spaceborne wide-field telescope monitoring the Earth's nighttime atmosphere with the objective of recording the ultraviolet light from tracks initiated by ultra-high energy cosmic rays. Through a series of stratospheric balloon flights performed by the French Space Agency CNES, EUSO-BALLOON will serve as a test-bench for the key technologies of JEM-EUSO. EUSO-BALLOON shall perform an end-to-end test of all subsystems and components, and prove the global detection chain while improving our knowledge of the atmospheric and terrestrial ultraviolet background. The ballooninstrument also has the potential to detect for the first time UV-light generated by atmospheric airshower from above, marking a key milestone in the development of UHECR science, and paving the way for any future large scale, space-based ultra-high energy cosmic ray observatory.
\end{abstract}

Key words : extragalactic cosmic-rays, ultra-high energy cosmic rays, balloon-borne instrumentation

\section{Context of the balloon program EUSO-BALLOON}


EUSO-BALLOON is a prototype of JEM-EUSO, the Extreme Universe Space Observatory on-board the Japanese Experiment Module of the International Space Station (ISS). JEM-EUSO is designed to identify the astrophysical origin and nature of the ultra high-energy cosmic rays (UHECRs), the most energetic particles known in the Universe. JEM-EUSO will monitor a gigantic detection volume by looking downward to the Earth's atmosphere from the ISS, observing the UV fluorescence light of UHECR-induced Extensive Air Showers (EAS). The two ton space instrument features Fresnel optics with a large field of view $\left( \pm 30^{\circ}\right)$, and a focal detector surface of $\sim 5000$ multi-anode photomultipliers - i.e. more than 300000 pixels. The mission consortium includes 13 countries and is lead by RIKEN (Japan), in coordination with the Japanese space agency (JAXA). A comprehensive description of JEM-EUSO is given by Ebisuzaki (2012).

EUSO-BALLOON is developed by the JEM-EUSO consortium as a demonstrator for the technologies and methods featured in the space instrument. Since the JEM-EUSO's observation of UHECR-induced EAS is based on the detection of an UV light track (fluorescence emission of Nitrogen molecules excited by collisions with shower particles), EUSO-BALLOON is an imaging UV telescope as well. The balloon-borne instrument points towards the nadir from a float altitude of about $40 \mathrm{~km}$. With its Fresnel Optics and Photo-Detector Module, the instrument monitors a $12 \times 12^{\circ}$ wide field of view in a wavelength range between 290 and $430 \mathrm{~nm}$, at a rate of 400'000 frames/sec. The EUSO-BALLOON mission has been proposed by a collaboration of three French laboratories (APC, IRAP and LAL) involved in the international JEM-EUSO consortium. The instrument is presently built by various institutes of the entire JEM-EUSO collaboration. Balloon flights will be performed by the balloon division of the French Space Agency CNES, a first flight is scheduled for 2014.

\section{Objectives of the balloon flights}

EUSO-BALLOON will serve as a test-bench for the JEM-EUSO mission as well as any future mission dedicated to the observation of extensive air shower from space. The following objectives shall be attained in a series of balloon flights :

\section{A) technology demonstrator}

EUSO-BALLOON is a full scale end-to-end test of all the key technologies and instrumentation of JEM-EUSO. Particularly, crucial issues that will benefit from the balloon flights include the HV power supplies, the HV switches (HV relays commuting the HV in case a city or a bright atmospheric event comes into the FOV and on a pixel), the Front-End Electronics (including the ASICs and FPGA), the on-board hardware and software algorithms involved in the triggering and recognition of cosmic-ray initiated air showers.

\section{B) data acquisition and background study}

Although the physics and the detection technique of EAS through ultraviolet light (UV) emission is well established and daily used in ground based detectors (see e.g. The Pierre Auger Collaboration, 2010; Kawai et al. 2008), their observation from space has never been performed. Since JEM-EUSO uses the Earth's atmosphere to observe UV (300-400 nm) fluorescence tracks and Cherenkov reflections from EAS, the observations will be sensitive to the variation of the background sources in the UV range. Whereas a number of background measurements have been performed, even from space, no focusing instruments have been employed so far and, most importantly, spatial resolutions were extremely low, i.e. the "pixel size" was much larger. Large localized background signals could have been washed out by the integration over a large surface, and likewise, possible temporal variations on small scales were not observable, and thus went unconstrained.

Observing EAS from space - for the first time - will confirm the feasibility of the technique and 
provide valuable data for JEM-EUSO, and all future space-borne UHECR experiments.

The main objectives are thus:

- experimental confirmation of the effective background below $40 \mathrm{~km}$ observed with a pixel size on ground representative for JEM-EUSO (175 m x $175 \mathrm{~m}$ in a +/-6 $6^{\circ}$ field of view).

- acquisition of $2.5 \mu \mathrm{sec}$ frames of UV signal and background (in a format similar to JEM-EUSO) from a balloon-borne gondola.

- testing of observational modes and switching algorithms.

- testing/optimization of the trigger algorithms with real observations and varying background.

- testing/optimization of the trigger algorithms with real observations above various ground surfaces.

- testing of the acquisition capability of the infrared-camera.

\section{C) pioneering mission for JEM-EUSO}

A "bonus objective" of the EUSO-BALLOON is the detection of air-showers by looking downward from the edge of space to- the threshold for these obviously rare EAS events being of the order of a few $10^{18} \mathrm{eV}$. The detection of LASER-induced events from ballon-altitudes will provide a proof of principle and a way to calibrate the threshold / sensitivity.

\section{Payload Overview}

The general layout of EUSO-BALLOON is shown in Figure 1, its main components are the optical bench and the instrument booth. An electronic block diagram of the entire instrument, summarizing all subsystems and components is shown in Figure 2. The development of all components and subassembly items is based on similar JEM-EUSO components and sub-assemblies. The total mass of the payload is about $320 \mathrm{~kg}$; two battery packs will maintain constant power of $225 \mathrm{~W}$ during 24 hours of flight (which is more than enough for a first flight that is to last one night only).

The optical bench contains three Fresnel lenses made from $8 \mathrm{~mm}$ thick PMMA (UV transmitting polymethyl-methacrylate) with a front surface of $100 \times 100 \mathrm{~cm}$ each. The EUSO-BALLOON optics has been designed to resemble the JEM-EUSO optics (i.e. three Fresnel lenses) : it is dimensioned to produce a RMS spot size smaller than the pixel size of the detector (i.e. $2.85 \mathrm{~mm}$ ) and keep the background rate per pixel comparable to the one anticipated for JEM-EUSO (i.e. roughly $2 \pm 1$ photoelectrons per pixel and GTU of $2.5 \mu \mathrm{sec}$ ). A ray tracing diagram of the optical bench is shown in figure 3 : whereas L1 and L3 are aspherical Fresnel Lenses with focal lengths of $258.56 \mathrm{~cm}$ and 60.02 $\mathrm{cm}$, respectively, L2 is a diffractive lens with focal of $385.69 \mathrm{~cm}$ (focal lengths are reference values only, single lenses are not producing stigmatic images). Within the optical bench, the position of L1 and L2 can be adjusted along the optical (z-)axis. Together with the 15 x $15 \mathrm{~cm}$ focal plane detector (PDM, see below) the optics provides a field of view of $\pm 6^{\circ}$.

The instrument booth is made as a watertight capsule ${ }^{1}$ using the third Fresnel lens (L3) as a porthole. Besides of the focal plane detector (PDM) and associated electronics (DP) which are described below the instrument booth houses the telemetry system (SIREN), CNES specific instrumentation (ICDV, Hub), and two battery-packs.

The Photo-Detector Module (PDM) is schematically shown in Figure 4. It consists of

1 EUSO-BALLOON will eventually observe above open water; the payload has deliberately been designed to protect all sensitive equipement on a water-landing 
- 36 MAPMT, organized in

- 9 Elementary Cells (EC) containing four ASIC's each,

- Cockroft Walton High-Voltage generators,

- High-Voltage switches, and a

- PDM board including an FPGA.

UV light collected by the telescope is focused onto - and detected - by the PDM, which is composed of 36 MAPMT (Hamamatsu M64 multi-anode photomultipliers) containing 64 anodes each. The PDM is organized in 3x3 Elementary Cells (EC) which in turn are composed of 2x2 MAPMTs. A UV colour glass filter is bonded to the window of the MAPMT with optical glue. The filter (SCHOTT BG3 with anti-reflection coating) transmits UV light in a band between 290 and $430 \mathrm{~nm}$. The EC unit supplies the voltages produced by the High Voltage Power Supply to each of its MAPMTs, collects the signals from their anodes and transmits them to the ASIC for processing.

Each of the 2304 pixels (anodes) in the PDM is sensitive to single photons, and features a dynamic range of -a with 6 orders of magnitude thanks to an adaptive gain. The dynodes are driven by Cockroft Walton High-Voltage generators. In order to protect the photodetectors against highly luminous events (lightnings, eity-lights etc.), custom made High-Voltage switches are capable of reducing the gain in a few microseconds.

The analogue signal from the MAPMTs anodes is continuously digitizes and processed by the FrontEnd Electronics based on the "SPACIROC" ASIC (Spatial Photomultiplier Array Counting and Integrating Readout Chip, see Ahmad 2011). The ASIC features a single photo-electron (SPE) mode as well a charge integration (KI) mode in order to measure the intensity of the photon flux (charge to time conversion). Data acquisition and readout is performed within a defined time slot called Gate Time Unit (GTU=2.5 $\mu$ s). This is fast enough to observe the speed-of-light phenomena in EAS.

The output signals from the four ASICs of an EC unit are transmitted to the PDM board which can handle all 9 EC units. The hardware of the PDM board electronics includes an FPGA (Field Programmable Gate Array, the present baseline is the Virtex6 XC6VLX240T), which performs a firstlevel trigger algorithm (persistency track trigger). A shower candidate is triggered if in a box of $3 \times 3$ pixels there is an excess of signal above expected background fluctuations for few consecutive GTUs. The parameters will be adapted on flight as a function of the average background level.

The Digital Processor (DP) is composed of the following sub-assemblies :

- Control Cluster Board (CCB),

- CPU,

- Data storage (DST),

- Housekeeping system (HK),

- Clock Board (CLKB),

- GPS receiver (GPSR),

- Data Processor Power Supply (DP-LVPS).

The CCB is developed around a Xilinx Virtex-4 FX-60, it collects the data from the PDM board, processes and classifies the received data, and performs a second level trigger filtering. The DP is then tagging the events with their arrival time (UTC) and payload position (GPS), it manages the Mass Memory for data storage, measures the operating- and dead-time of the instrument, provides signals for time synchronization of the event, performs housekeeping monitoring, and handles the interface to telecommands and telemetry system.

Table 1 : Comparison of the principle characteristics between JEM-EUSO and EUSO-

BALLOON. The field of view of EUSO-BALLOON - and hence its pixel size - has 
been dimensioned to see a level of nightglow background that is comparable to the one expected for JEM-EUSO.

\begin{tabular}{lll}
\hline & JEM-EUSO & EUSO-BALLOON \\
\hline Number of PDM & 143 & 1 \\
Flight Altitude [km] & 420 & 40 \\
Diameter of Optics [km] & 2.5 & 1 \\
Field of View / PDM & $3.8^{\circ}$ & $12^{\circ}$ \\
PDM@ground [km] & 28.2 & 8.4 \\
Field of View / pixel & $0.08^{\circ}$ & $0.25^{\circ}$ \\
Pixel@ground [km] & 0.580 & 0.175 \\
Signal Ratio w/r to JEM-EUSO & 1 & 17.6 \\
BG Ratio w/r to JEM-EUSO & 1 & $0.9-1.8$ \\
S/NN w/r to JEM-EUSO & 1 & $20-10$ \\
Threshold Energy [eV] & $3 \cdot 10^{19} 19$ & $1.5-3 \cdot 10^{18} 18$ \\
\hline
\end{tabular}

An event selected by the two trigger levels roughly represents $330 \mathrm{kB}$ of data. Since only a limited data rate can be transmitted to the ground through CNES' new NOSICA telemetry system, all data will be systematically stored on board. The mass storage is composed by two Solid-State Drive (SSD) each one with 1 TB capacity operating in disks fault-tolerant mode RAID-1 (Redundant Array of Independent Disks).

Balloon operation : During a first flight the payload will operate in nadir pointing mode, the spin rate will be determined by the natural azimuthal oscillations of the flight train. For later flights, the inclination of the pointing axis will be controlled between 0 and $30^{\circ} \mathrm{w} / \mathrm{r}$ to the nadir and an azimuth motor will provide the possibility to perform revolutions with a spin rate of up to $3 \mathrm{rpm}$. Performing azimuthal revolutions will simulate a groundspeed comparable to the $\sim 7 \mathrm{~km} / \mathrm{s}$ of the space-station, permitting a full scale test of the HV-switches : i.e. switching MAPMT voltages on/off within a few microseconds, as artificial and other light sources cross the field of view of the instrument.

As the first balloon flight shall take place from a new CNES launch base in Timmins, Canada (lat $48.5^{\circ} \mathrm{N}$ ) a number of different groundcovers will be overflown, including various types of soil and vegetation, water, urban and industrial areas, and - very likely - clouds. EUSO-BALLOON should therefore be able to measure a representative variety of background conditions.

Performance : The test of all key components and sub-assembly items foreseen in JEM-EUSO is the first (A-level) objective of EUSO-BALLOON. A second prime objective (B-level) is measuring a relevant background and testing the trigger schemes by coping with variable background conditions. This goal has been the principal driver for determining the pixel size, and hence the global Field of View of EUSO-BALLOON. The EUSO Simulation and Analysis Framework (ESAF) has been adapted to simulate the response of the instrument (see e.g. Adams 2013). The configuration used for JEM-EUSO has been modified scaling for the altitude of the instrument, changing the surface parameterization, introducing the new optical system and field of view (see Table 1). 
Table 2 : EUSO-BALLOON : Responsibilities in the procurement and delivery of the various components of the EUSO Balloon instrument. For full names and addresses of the contributing institutions see the indexed list on the title page.

\begin{tabular}{|c|c|c|c|}
\hline Component & Sub-Assembly & Lab / University & Country \\
\hline \multirow{3}{*}{ Telescope } & Fresnel Lenses & RIKEN $^{14}$ & Japan \\
\hline & Lens Fitting & \multirow{2}{*}{ IRAP $^{1}$} & \multirow{2}{*}{ France } \\
\hline & Telescope Structure / Gondola & & \\
\hline \multirow{7}{*}{ PDM } & Multi-Anode Photo-Multipliers & RIKEN $^{14}$ & Japan \\
\hline & ASIC & $\mathrm{LAL}^{2}$ & France \\
\hline & Elementary Cell & $\mathrm{LAL}^{2} / \mathrm{KIT}^{6}$ & France / Germany \\
\hline & HV Power Supply and HV Switches & $\mathrm{NCBJ}^{17} / \mathrm{APC}^{3}$ & Poland / France \\
\hline & PDM Board & EWHA $^{15}$ & South Korea \\
\hline & PDM Low Power Supply & UNAM $^{16}$ & Mexico \\
\hline & PDM Structure & INFN Frascati $^{8}$ & Italy \\
\hline \multirow{7}{*}{$\begin{array}{l}\text { Digital } \\
\text { Processor }\end{array}$} & CCB Data Processor & IAAT $^{5}$ & Germany \\
\hline & Main processing unit & \multirow{3}{*}{ INFN Napoli ${ }^{9,10}$} & \multirow{3}{*}{ Italy } \\
\hline & Data Storage & & \\
\hline & Clock Generator & & \\
\hline & Trigger software & Uni-INFN Torino $^{13}$ & Italy \\
\hline & House Keeping Board & UNAM $^{16}$ & Mexico \\
\hline & Low Voltage Power Supply & UNAM $^{16}$ & Mexico \\
\hline Battery Pack & Batteries and Controller & RIKEN $^{14} /$ IRAP $^{1}$ & Japan/France \\
\hline IR-CAM & IR Camera & $\mathrm{UAH}$ & Spain \\
\hline
\end{tabular}

While the detection of Extensive Air Showers was not amongst the initial objectives for EUSOBALLOON, the simulation showed that the instrument was able to detect and image Extensive Air Showers with energies above $10^{18} \mathrm{eV}$. This threshold energy arises from the background estimate reported by Sakaki et al. 2008. A first analysis indicates that $0.2-0.3$ events/night $\left(\mathrm{E}>2.1 \underline{\underline{x}}=10^{18} \mathrm{eV}\right)$ are expected during $10 \mathrm{~h}$ of flight. The uncertainty in the estimation assumes also the presence of a certain cloud fraction. Anyway, the objective of the first flight is to focus on objectives a) and b). (see figure 5). A clear detection requires long duration balloon flights - this is foreseen for subsequent launches and has become a further objective (C-level) for EUSO-BALLOON.

In order to monitor the actual cloud covers, a co-aligned IR camera will observe the field of view of the main instrument (similar to the one used on JEM-EUSO, see e.g. Morales de los Rios, 2011). A possible option to create signal-type events is to use an on-board laser for simulating air-showers within the field of view.

\section{Project Organization and Status}

EUSO-BALLOON is designed and built entirely within the JEM-EUSO collaboration. As its pathfinder, EUSO-BALLOON is identical (PDM, triggers etc.) or similar (optics) to the main mission. All relevant institutions and international partners within the JEM-EUSO collaboration contribute to the instrument (Table 2) according to their corresponding tasks and responsibilities in JEM-EUSO. 
A ground based prototype, very similar to EUSO-BALLON, has recently been integrated at RIKEN, Japan and installed on the site of Telescope Array (TA) in Utah, USA. It is designed to cross calibrate the instrument with TA Fluorescence Detector through noise background comparison and during Lidar or electron beam shots.

EUSO-BALLOON is a mission of the French Space Agency CNES, lead under the responsibility of the French team, which acts in coordination with the JEM-EUSO management. As this article is submitted, the Critical Design Review at CNES has been held, a qualification model of the entire electronics chain has shown to operate, and the Fresnel optics is under fabrication : the EUSOBALLOON project is on track for its first balloon flight in 2014 !

Acknowledgement: We acknowledge strong support from the French Space Agency CNES.

\section{References}

Adams, J. H. , 2013, An Evaluation of the Exposure in Nadir Observation of the JEM-EUSO Mission, Astroparticle Physics 44, 76-90

Ahmad, S., 2011, SPACIROC: A Front-End Readout ASIC for the JEM-EUSO observatory, proc. IEEE Nuclear Science Symposium and Medical Imaging Conference (NSS/MIC), p. 699

Ebisuzaki T., The JEM-EUSO mission, 2012, Adv Space Res, these proceedings

Kawai H, et al., Nucle. Phys. B Proc. Suppl. 175-176 , (2008) 221

Morales de los Rios, J.-A., 2011, The IR-Camera of the JEM-EUSO Space Observatory, Proc. 32nd ICRC, Vol. 11, p. 466

Sakaki, N. et al., 2005, Balloon-borne measurement of UV nightglow and clouds for the JEM-EUSO mission, Proc. 30th ICRC, Vol. 5, p. 965

The Pierre Auger Collaboration, Nucl. Instrum. Meth., 2010, A620 : 227-251. 


\section{Figure Captions}

Figure 1 : Schematic of the instrument, composed of an optical bench and a watertight instrumentbooth.

Fig. 2 : Functional Block Diagram of the EUSO-BALLOON Instrument

Fig. 3 : Ray tracing diagram of the EUSO-BALLOON optics - the Fresnel lenses L1, L2, L3 have a surface of $1 \mathrm{x} 1 \mathrm{~m}$, they are made from $8 \mathrm{~mm}$ thick PMMA

Fig. 4 : Schematic view of the Photo-Detector Module (PDM) : the MAPMTs are covered with UV filters; four MAPMT's form an Elementary Cell (EC). The PDM includes 9 ECs, 6 EC-boards and a PDM board.

Fig. 5 : Preliminary estimate of the number of EAS detected during a $10 \mathrm{~h}$ balloon flight. The three curves represent different assumptions of background and cosmic ray flux. 


\section{Figure 1}

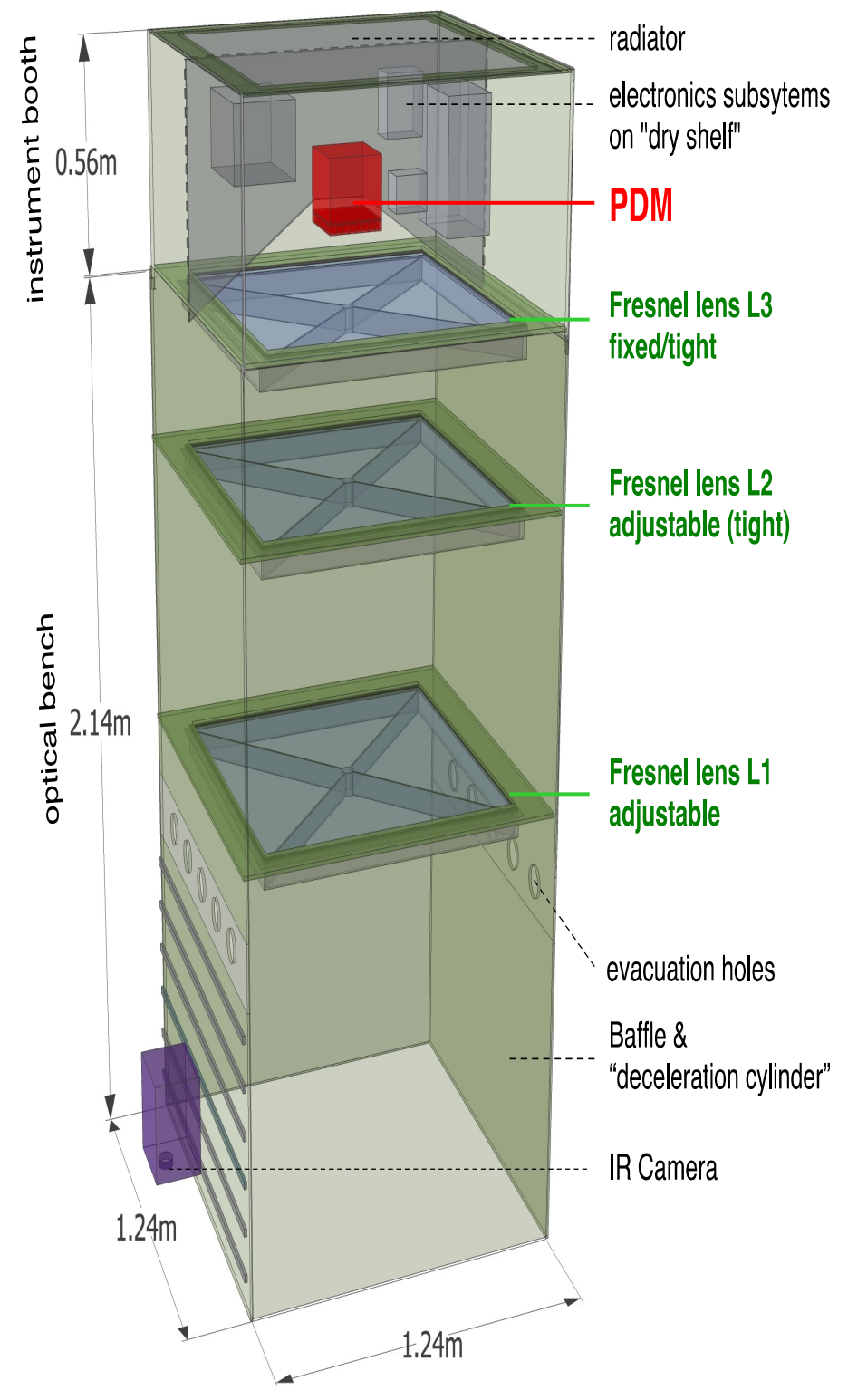


Figure 2

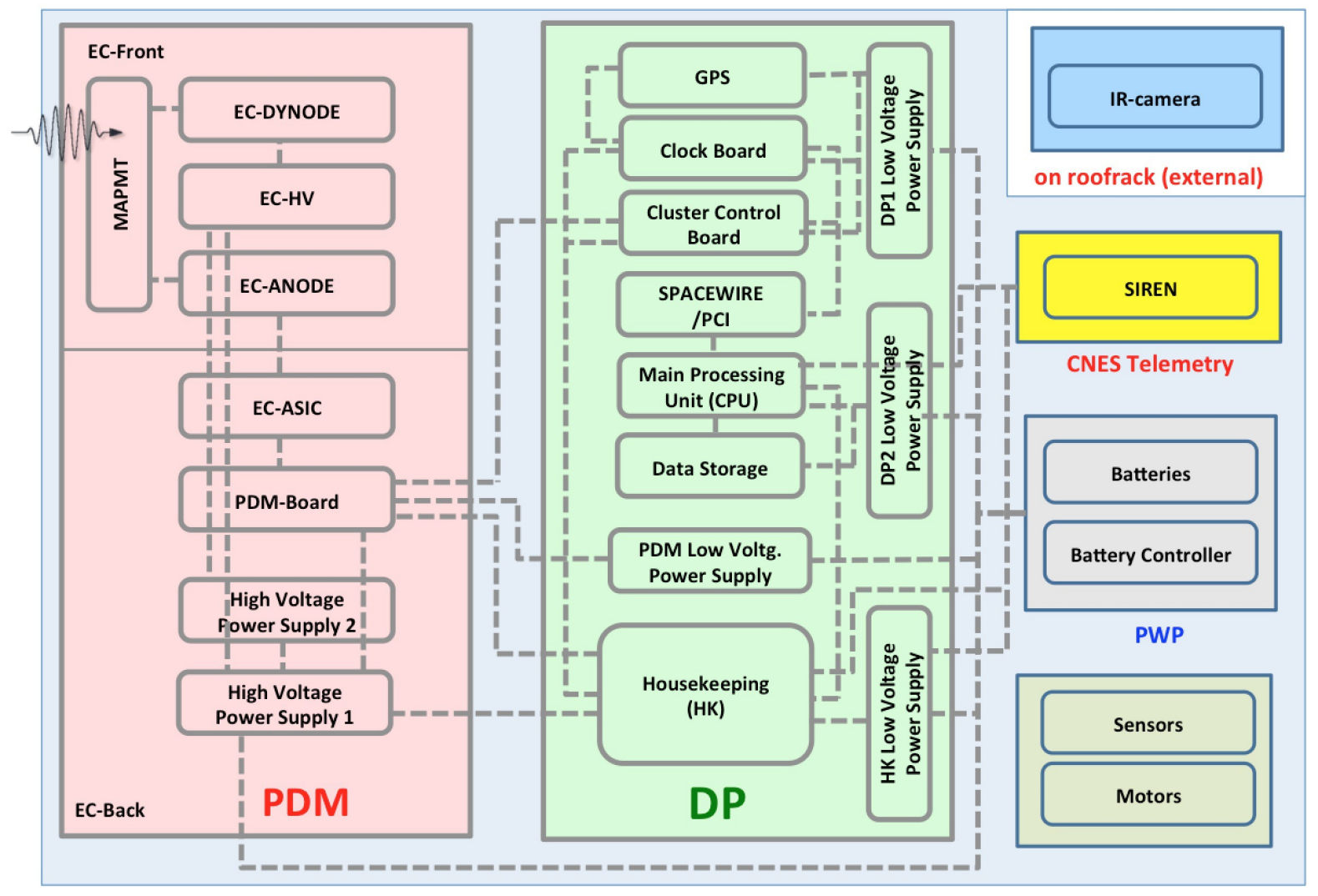


Figure 3

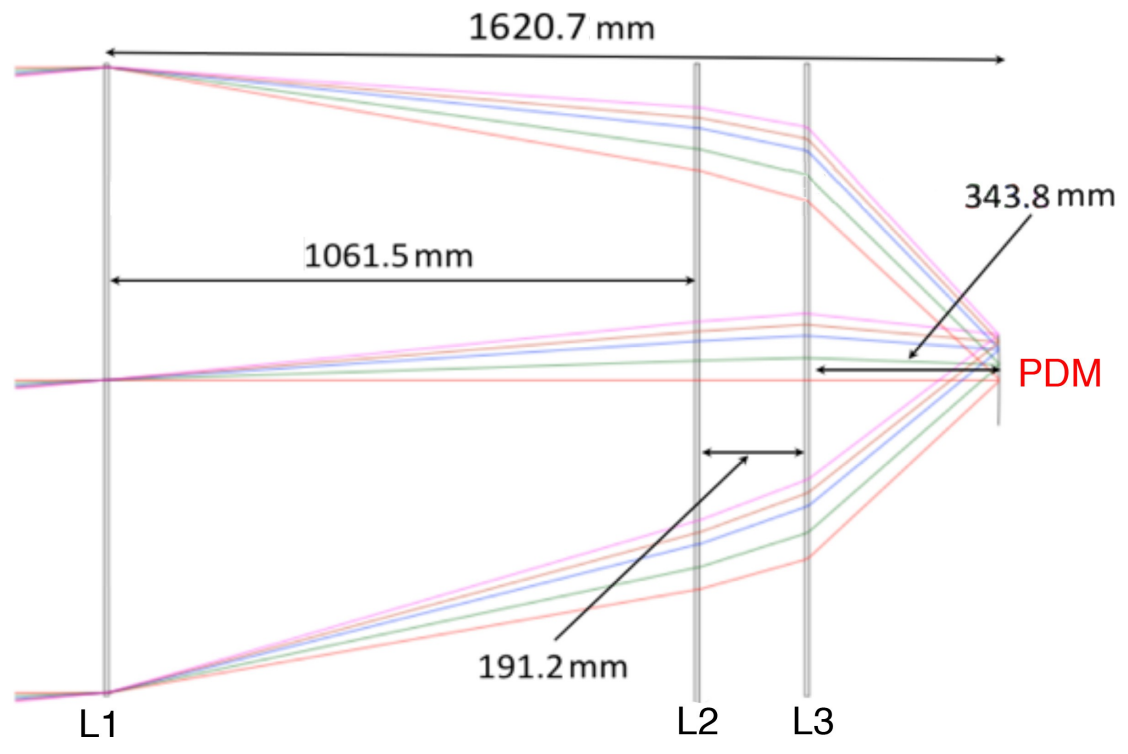


Figure 4
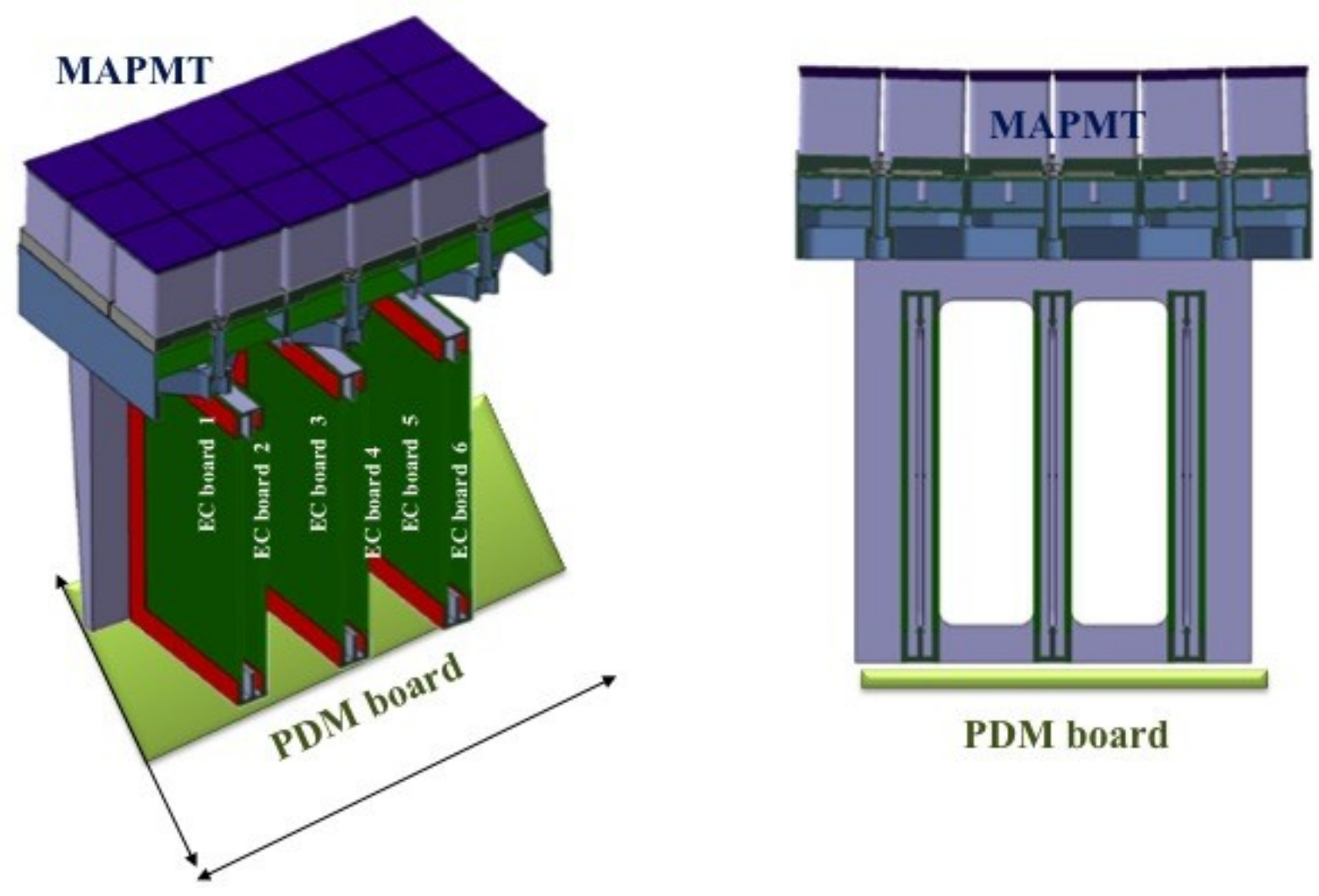
Figure 5

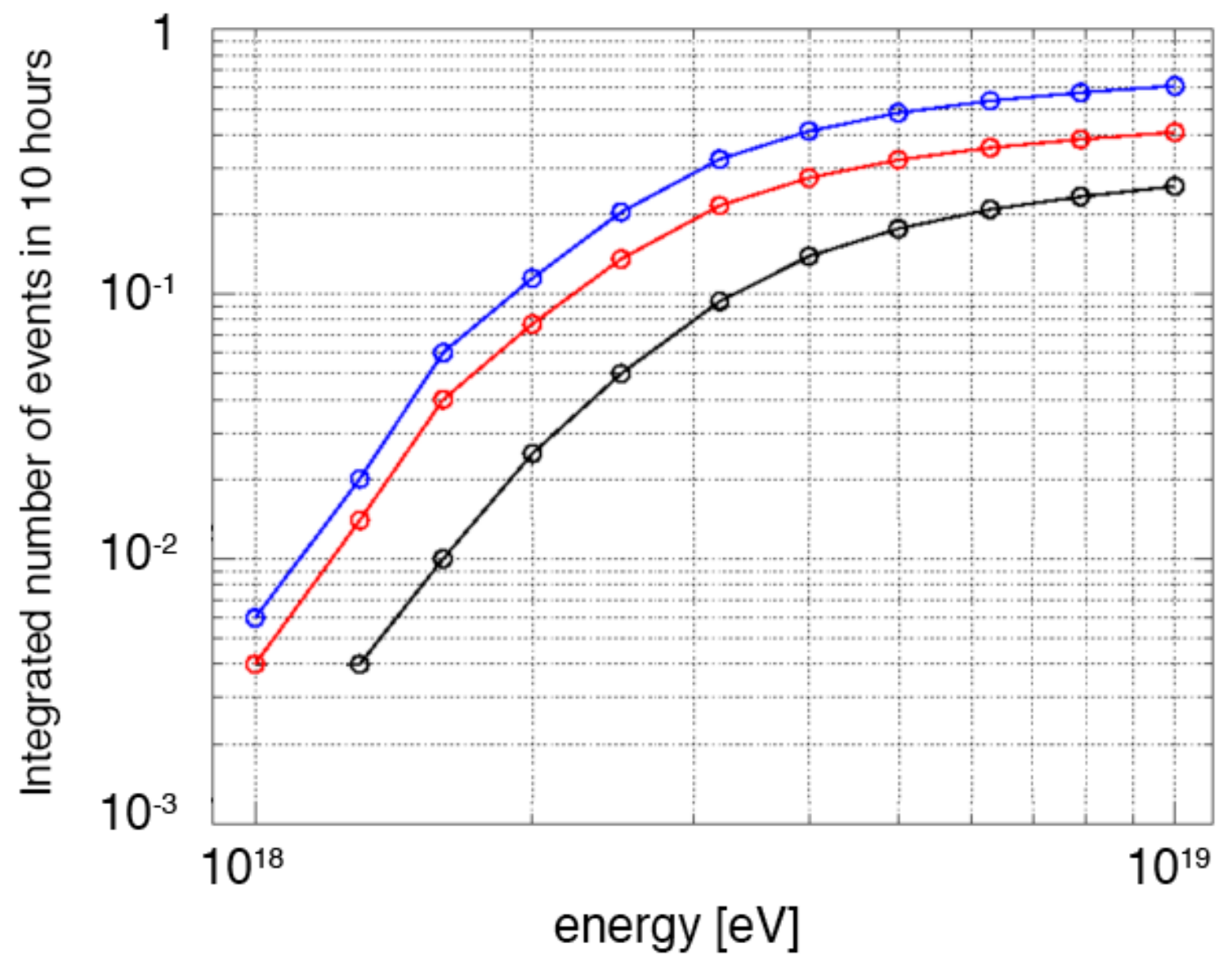

